\title{
Comparison of topical clobetasol propionate $0.05 \%$ and topical tacrolimus $0.1 \%$ in the treatment of cutaneous lichen planus
}

\author{
Ezgi Özkur ${ }^{1}$, Esra Koku Aksu², Mehmet Salih Gürel², Sevil Savaş ${ }^{3}$ \\ ${ }^{1}$ Şişli Etfal Training and Research Hospital, Health Science University, Istanbul, Turkey \\ 2Istanbul Training and Research Hospital, Health Science University, Istanbul, Turkey \\ ${ }^{3}$ Sultan abdülhamithan Training and Research Hospital, Health Science University, Istanbul, Turkey \\ Adv Dermatol Allergol 2019; XXXVI (6): 722-726 \\ DOI: https://doi.org/10.5114/ada.2019.91423
}

\begin{abstract}
Introduction: The effectiveness of topical tacrolimus in the treatment of oral and genital lichen planus has been verified in many randomized studies; however, there are only few case reports in treatment of cutaneous lichen planus (CLP).

Aim: We sought to compare the safety and efficacy of topical clobetasol propionate and tacrolimus ointment in the treatment of CLP.

Material and methods: Retrospective analysis of patient files was performed. We enrolled patients who were diagnosed with CLP and treated with topical tacrolimus $0.1 \%$ or topical clobetasol propionate $0.05 \%$. Visual Analogue Scale (VAS) scores of pigmentation and pruritus, clinical response, laboratory data and adverse effects were obtained from medical records.

Results: A total of 27 patients were included in the clobetasol group and 23 patients in the tacrolimus group. Both groups showed an improvement in VAS scores regarding pruritus and pigmentation but a statistically significant difference was observed in the clobetasol group $(p<0.05)$. At week 12 , a complete response was observed in $63 \%$ $(n=17)$ of the clobetasol and $26 \%(n=6)$ of the tacrolimus group.

Conclusions: In our study, both treatments were found effective in the treatment of CLP but clobetasol propionate was more effective. However tacrolimus may be preferred before topical corticosteroids for lesions on the face, neck, and intertriginous regions of the body, which are sensitive to the cutaneous adverse effects of topical corticosteroids. Our study may be one of the first studies to compare the effects of topical clobetasol and tacrolimus ointment in the management of CLP.
\end{abstract}

Key words: lichen planus, tacrolimus, clobetasol propionate.

\section{Introduction}

Cutaneous lichen planus (CLP) is a papulosquamous eruption of skin which is characterized by violaceous papules and plaques and mostly located on the extremities but may occur in the other parts of the body or oral and genital mucosa. CLP is a benign dermatological disease, however, patients are often troubled by pruritus and pigmentation. The current first-line therapy is topical corticosteroids [1]. Although treatment with topical corticosteroids is effective, in most trials, quality levels of the body of evidence range from very low to moderate, so more well-designed studies are needed to inves- tigate the efficacy of topical corticosteroids [2]. Adverse effects of topical steroids are well known and include: thinning of skin, infections, striae, systemic absorption, and hypothalamic-pituitary axis suppression. Therefore, a different treatment regimen for CLP that does not rely on corticosteroids may be beneficial.

Topical tacrolimus and pimecrolimus are effective topical calcineurin inhibitors (TCI), which are anti-inflammatory agents that allow treatment of inflammatory dermatoses without the adverse effects of topical corticosteroids. They are already approved for the treatment of atopic dermatitis in patients older than 2 years,

\footnotetext{
Address for correspondence: Ezgi Özkur MD, Şişli Etfal Training and Research Hospital, Health Science University, Istanbul, Turkey, e-mail: ezgierdal@hotmail.com

Received: 2.05.2018, accepted: 18.07.2018. 
but there are many small series and case reports reporting successful use of TCls in various other skin diseases [3-6]. Double-blind and open studies have shown favourable results with topical tacrolimus and pimecrolimus in mucosal lichen planus [7-10].

\section{Aim}

This retrospective study aimed to compare the effectiveness of topical clobetasol propionate $0.05 \%$ and topical tacrolimus $0.1 \%$ in patients with CLP.

\section{Material and methods}

Overall, 50 patients (27 patients in the clobetasol group and 23 patients in the tacrolimus group) who met the inclusion criteria were enrolled to this case series study retrospectively. Patients who were diagnosed as CLP clinically and histopathologically and who were prescribed topical tacrolimus $0.1 \%$ or topical clobetasol propionate $0.05 \%$ ointment were enrolled in the study and compared.

CLP was histologically confirmed in all patients and diagnosis was made with the presence of a band-like cellular infiltration comprising mainly lymphocytes and signs of basal cell layer degeneration and Civatte bodies. Patients who had already received or were receiving treatment for CLP with anything other than topical tacrolimus $0.1 \%$ or topical clobetasol propionate $0.05 \%$ were excluded. Patients receiving systemic immunosuppressants or received them in the last year were also excluded. Demographic features, duration of disease, LP subtype, distribution of lesions, thickness and pigmentation scores, visual analogue scale (VAS) scores, laboratory values, adverse effects and all patient's week 4, 8, and 12 follow-up notes were retrieved from the database.
Lesions were assessed by the patients themselves with regard to pigmentation of lesions and severity of pruritus at every visit using a VAS with a range of 0-10 with higher numbers indicating worse outcomes. At week 12, patients were classified according to the response to the treatment (elimination of the pruritus, elevation and erythema of the lesions), as complete response (more than 90\%), partial response (50-90\%), weak response (20-50\%), and no response (less than 20\%).

The study was performed according to the Helsinki declaration and an ethics committee's permission was obtained (on 07/01/2016, number: 23521).

\section{Statistical analysis}

Data analyses were performed using statistical software SPSS 15.0. Descriptive statistics are given as numbers and percentages for categorical variables, and averages and standard deviations for numeric variables. Comparisons between two independent groups were made using Student's $t$-test when numeric variables fulfilled the condition of normal distribution, and the MannWhitney $U$ test when they were not normally distributed. Comparisons of two dependent groups were made using the paired samples $t$-test when the differences of variables were normally distributed, and the Wilcoxon test when this criterion was not fulfilled. Under the circumstances where conditions could not be fulfilled, Monte Carlo simulation was applied. The statistical $\alpha$ (level of significance) level was accepted as $p<0.05$.

\section{Results}

Patients' demographic and disease characteristics are summarized in Table 1. There were no statistically signifi-

Table 1. Baseline demographics and medical information

\begin{tabular}{|c|c|c|c|c|}
\hline Parameter & & Tacrolimus $(n=23)$ & Clobetasol propionate $(n=27)$ & $P$-value \\
\hline Age, mean (min.-max.) [years] & & $47.7(24-69)$ & $50.6(21-68)$ & 0.640 \\
\hline \multirow[t]{2}{*}{ Sex, $n(\%)$} & Male & $8(34.8)$ & $10(37.0)$ & 0.869 \\
\hline & Female & $15(65.2)$ & $17(63.0)$ & \\
\hline Disease duration, mean \pm SD (min.-max.) [months] & & $9.4 \pm 15.1(1-72)$ & $7.1 \pm 6.5(1-24)$ & 0.914 \\
\hline \multirow[t]{4}{*}{ LP subtype, $n(\%)$} & Classical & $18(78.3)$ & $24(88.9)$ & 0.006 \\
\hline & Hypertrophic & $0(0.0)$ & $3(11.1)$ & \\
\hline & Actinic & $1(4.3)$ & $0(0.0)$ & \\
\hline & Pigmented & $4(17.4)$ & $0(0.0)$ & \\
\hline \multirow[t]{4}{*}{ Distribution of lesions, $n$ (\%) } & Face & $4(17.4)$ & $0(0.0)$ & \\
\hline & $\begin{array}{l}\text { Upper } \\
\text { extremities }\end{array}$ & $16(69.6)$ & $20(74.1)$ & 0.723 \\
\hline & $\begin{array}{l}\text { Lower } \\
\text { extremities }\end{array}$ & $10(43.5)$ & $20(74.1)$ & 0.028 \\
\hline & Trunk & 9 (39.1) & $9(33.3)$ & 0.670 \\
\hline
\end{tabular}



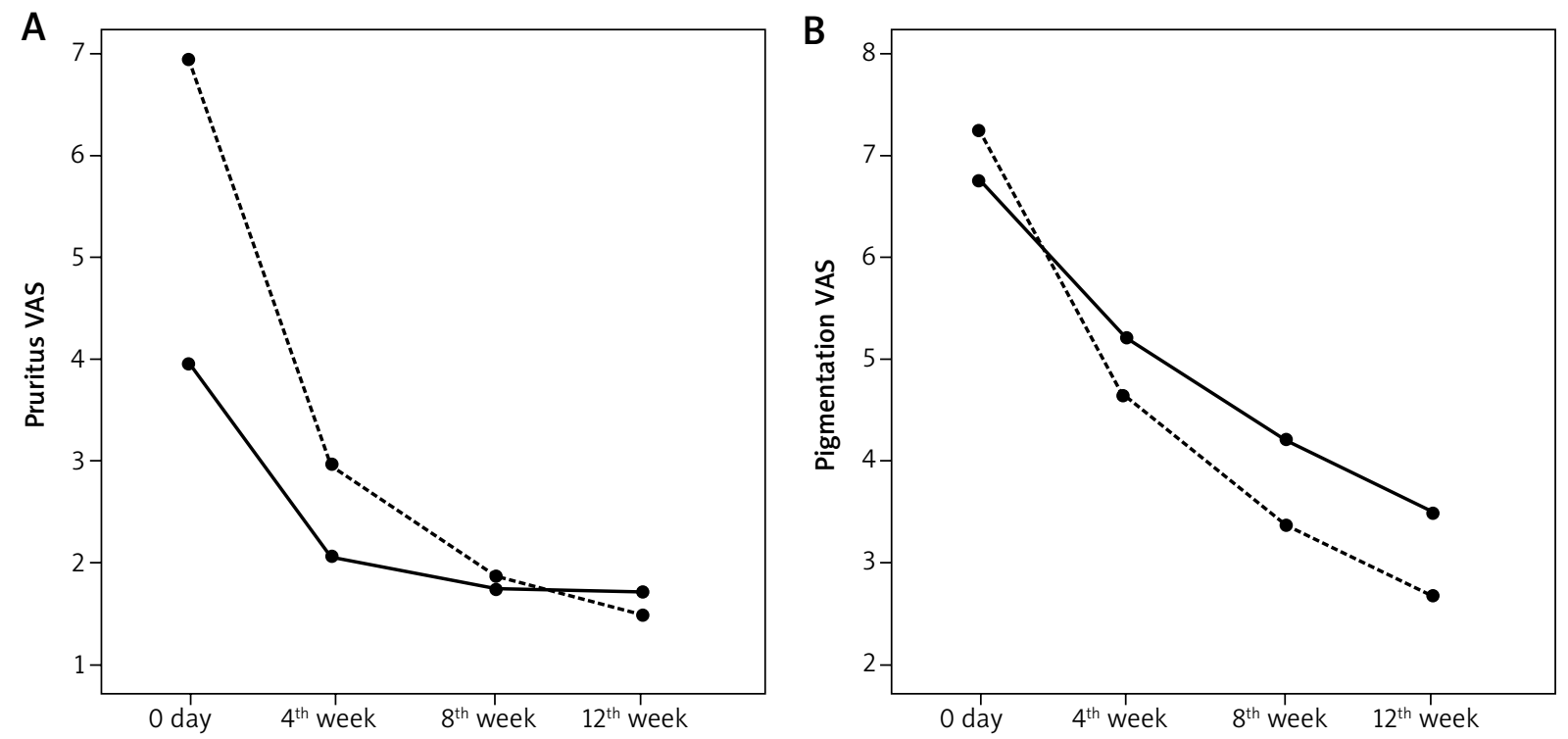

Figure 1. Patient VAS scores of pruritus (A) and pigmentation (B) by the treatment group. Both the clobetasol group (dashed line) and the tacrolimus group (solid line) showed an improvement

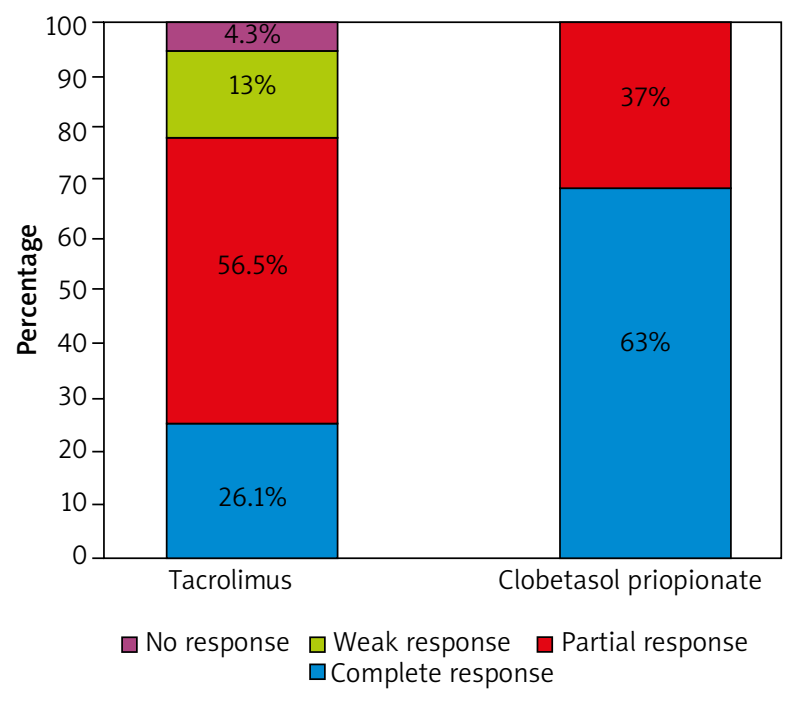

Figure 2. Week 12: clinical assessment

cant differences between two groups regarding age, sex or disease duration.

Both groups showed improvement in VAS scores (Figure 1). At week 12, complete response was $63 \%(n=17)$ in the clobetasol group and $26 \%(n=6)$ in the tacrolimus group (Figure 2). Ten patients responded partially in the clobetasol group and 13 patients in the tacrolimus group at the end of treatment. No treatment failure was observed in the clobetasol group.

There was a significant decrease in VAS scores regarding pruritus and pigmentation for both groups. However, there was a statistically higher improvement of VAS scores for pruritus and pigmentation found at week 12 with clobetasol $(p=0.004, p=0.038)$.

There was no statistically significant difference in terms of adverse cutaneous effects between the two groups. None of the patients in the tacrolimus group reported striae, telangiectasia or local infection. However, burning/pain symptoms were reported by 2 patients in the tacrolimus group and 5 patients in the clobetasol group in the overall treatment.

The mean lymphocyte count in peripheral blood did not decline after treatment in either group and statistical analysis of the counts of lymphocytes in peripheral blood before and after tacrolimus treatment showed no statistically significant difference $(p=0.879)$.

The mean overall clinical response at week 12 and disease duration showed no significant correlation in either treatment group ( $p=0.734, p=0.999$ ).

\section{Discussion}

Recently, topical tacrolimus and pimecrolimus have been shown to be effective in various inflammatory dermatoses without the adverse effects of topical corticosteroids. Oral lichen planus is one of the best studied off-label uses for topical calcineurin inhibitors [9-13]. In one headto-head study involving 32 patients, tacrolimus $0.1 \%$ ointment and clobetasol propionate $0.05 \%$ ointment showed significant improvement from baseline, and notably, tacrolimus was significantly better than clobetasol $(p<0.001)$ [14]. In another study of 30 patients with oral erosive/ulcerative lichen planus, tacrolimus $0.1 \%$ ointment was as effective as topical clobetasol propionate $0.05 \%$ ointment, both showing significant improvement from baseline [15]. In 

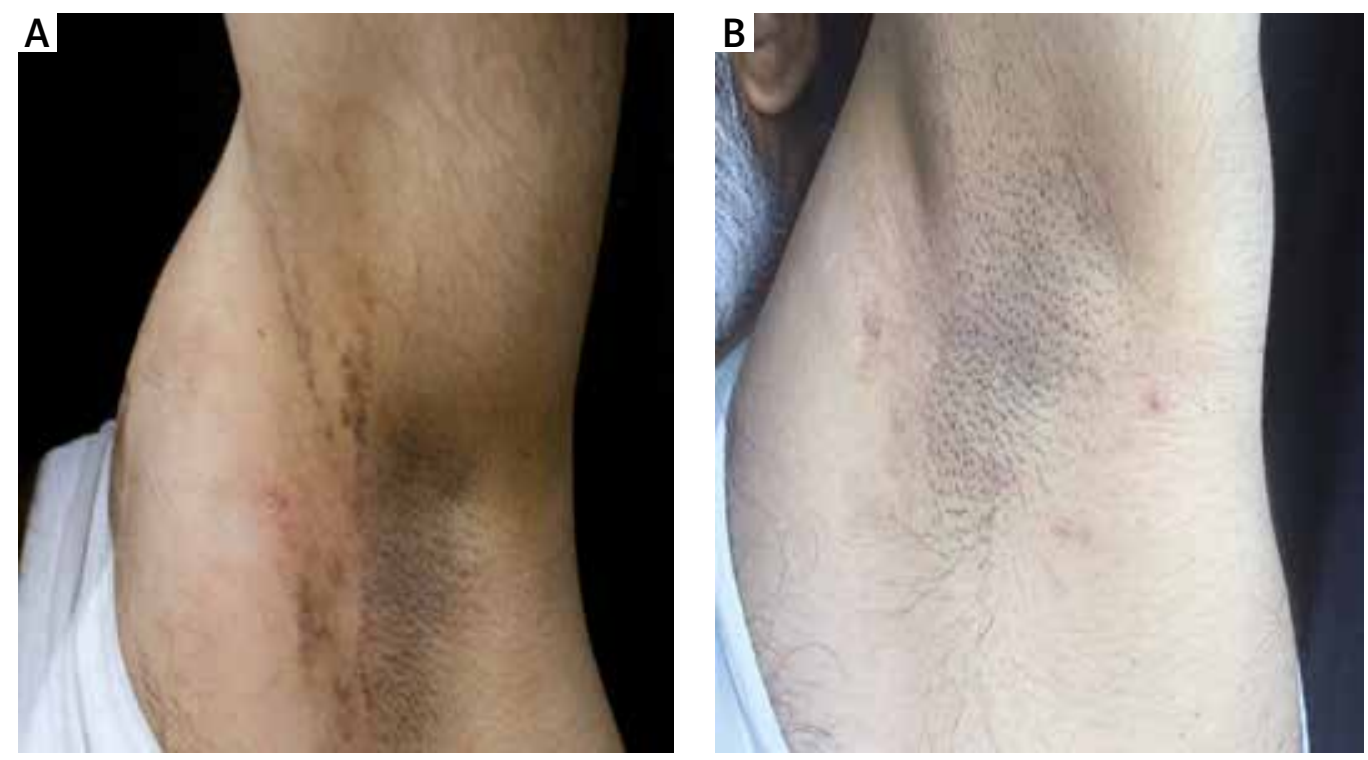

Figure 3. A patient with pigmented lichen planus before and after 12 weeks' treatment with topical tacrolimus $0.1 \%$
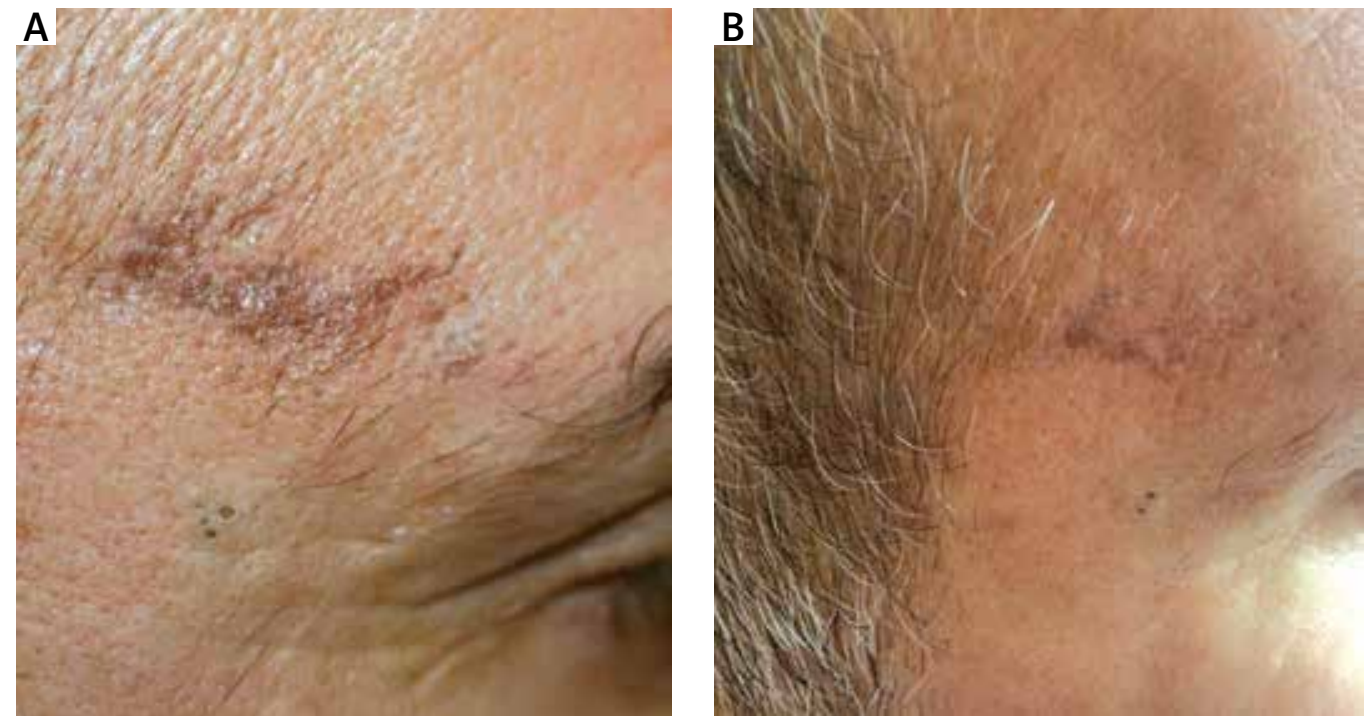

Figure 4. A patient with actinic lichen planus before and after 12 weeks' treatment with topical tacrolimus $0.1 \%$

a retrospective study, topical tacrolimus therapy improved lesions in 15 of 16 women with vulvar lichen planus [16]. Nevertheless, in one study, only 7 patients of 21 who were diagnosed with oral lichen planus and treated with topical $0.1 \%$ tacrolimus twice daily showed complete response at month 6; although it was a retrospective study, the authors suggested that the efficacy of topical tacrolimus was overestimated in daily practice [17].

Al-Mutairi et al. reported 13 patients with lichen planus pigmentosus who were treated with tacrolimus ointment and 7 (53.8\%) of them showed prominent lightening of the pigmentation within 12 weeks [18]. Our patients with pigmented and actinic lichen who were treated with tacrolimus planus also had a favourable response
(Figures 3 and 4). Pigmented and actinic lichen planus mostly occurs on light-exposed areas and needs longer treatments more than other forms. Therefore, topical tacrolimus may be preferred before topical corticosteroids for lesions on the face, which is very sensitive to longterm cutaneous adverse effects of topical corticosteroids.

A pilot study showed pimecrolimus cream $1 \%$ as an effective treatment regimen for severe lichen sclerosus in patients who were unsatisfactorily treated with topical corticosteroids previously [19]. Therefore, a longer treatment period in the tacrolimus group may have been more effective in our patients who showed partial, weak or no response. Also Paola et al. reported a successful outcome with pimecrolimus in hypertrophic genital lichen planus [20]. 
Tacrolimus has been studied too many times and has an acceptable safety profile. Simon et al. reported a decrease in lymphocyte counts in peripheral blood in 10 patients with atopic dermatitis who were treated with topical pimecrolimus [21]. In our study, the mean lymphocyte count in peripheral blood did not decline after treatment in either group.

In the literature, reported adverse effects of TCls are rare and include mostly itching or burning sensation [22]. Although more patients reported erythema and pruritus in the tacrolimus group, there was no statistical difference between the groups. Interestingly, burning and pain were reported by more patients in the clobetasol group. On the other hand, striae, telangiectasia, and local infections were reported only in the clobetasol group.

Limitations of our study were a small sample size, retrospective design and non-homogeneous distribution of specific LP types to treatment arms and non-usage of life quality indexes.

\section{Conclusions}

Both clobetasol and tacrolimus ointments are effective in the treatment of CLP. Our study suggests that topical clobetasol is more effective so it remains the most appropriate first-line therapy for CLP. However tacrolimus can be an option for patients who have failed therapy with topical corticosteroids or those who are contraindicated for the use of corticosteroids. It may be preferred before topical corticosteroids for lesions on the face, neck, and intertriginous regions of the body, which are sensitive to cutaneous adverse effects of topical corticosteroids.

\section{Conflict of interest}

The authors declare no conflict of interest.

\section{References}

1. Le Cleach L, Chosidow O. Clinical practice. Lichen planus. N Engl J Med 2012; 366: 723-32.

2. Antiga E, Caproni M, Parodi A, et al. Treatment of cutaneous lichen planus: an evidence based analysis of efficacy by the Italian Group for Cutaneous Immunopathology. G Ital Dermatol Venereol 2014; 149: 719-26.

3. Wollina $U$. The role of topical calcineurin inhibitors for skin diseases other than atopic dermatitis. Am J Clin Dermatol 2007; 8: 157-73.

4. Miller S. The effect of tacrolimus on lower extremity ulcers: a case study and review of the literature. Ostomy Wound Manage 2008; 54: 36-42.

5. Riano Arguelles A, Martino Gorbea R, Iglesias Zamora ME, Garatea Crelgo J. Topic tacrolimus, alternative treatment for oral erosive lichen planus resistant to steroids: a case report. Med Oral Patol Oral Cir Bucal 2006; 11: E462-6.

6. Al Johani KA, Hegarty AM, Porter SR, Fedele S. Calcineurin inhibitors in oral medicine. J Am Acad Dermatol 2009; 61: 829-40.
7. Lin AN. Innovative use of topical calcineurin inhibitors. Dermatol Clin 2010; 28: 535-45.

8. Vohra S, Singal A, Sharma SB. Clinical and serological efficacy of topical calcineurin inhibitors in oral lichen planus: a prospective randomized controlled trial. Int I Dermatol 2016; 55: 101-5.

9. Lodi G, Carrozzo M, Furness S, Thongprasom K. Interventions for treating oral lichen planus: a systematic review. Br J Dermatol 2012; 166: 938-47.

10. Garcia-Pola MJ, Gonzalez-Alvarez L, Garcia-Martin JM. Treatment of oral lichen planus. Systematic review and therapeutic guide. Med Clin (Barc) 2017; 149: 351-62.

11. Sotoodian B, Lo J, Lin A. Efficacy of topical calcineurin inhibitors in oral lichen planus. J Cutan Med Surg 2015; 19: 539-45.

12. Gisondi P, Ellis CN, Girolomoni G. Pimecrolimus in dermatology: atopic dermatitis and beyond. Int J Clin Pract 2005; 59 : 969-74.

13. Siponen M, Huuskonen L, Kallio-Pulkkinen S, et al. Topical tacrolimus, triamcinolone acetonide, and placebo in oral lichen planus: a pilot randomized controlled trial. Oral Dis 2017; 23: 660-8.

14. Corrocher G, Di Lorenzo G, Martinelli N, et al. Comparative effect of tacrolimus $0.1 \%$ ointment and clobetasol $0.05 \%$ ointment in patients with oral lichen planus. J Clin Periodontol 2008; 35: 244-9.

15. Radfar L, Wild RC, Suresh L. A comparative treatment study of topical tacrolimus and clobetasol in oral lichen planus. Oral Surg Oral Med Oral Pathol Oral Radiol Endod 2008; 105: 187-93.

16. Byrd JA, Davis MD, Rogers RS $3^{\text {rd }}$. Recalcitrant symptomatic vulvar lichen planus: response to topical tacrolimus. Arch Dermatol 2004; 140: 715-20.

17. Ribero S, Stieger M, Quaglino P, et al. Efficacy of topical tacrolimus for oral lichen planus: real-life experience in a retrospective cohort of patients with a review of the literature. J Eur Acad Dermatol Venereol 2015; 29: 1107-13.

18. Al-Mutairi N, El-Khalawany M. Clinicopathological characteristics of lichen planus pigmentosus and its response to tacrolimus ointment: an open label, non-randomized, prospective study. J Eur Acad Dermatol Venereol 2010; 24: 535-40.

19. Nissi R, Eriksen H, Risteli J, Niemimaa M. Pimecrolimus cream $1 \%$ in the treatment of lichen sclerosus. Gynecol Obstet Invest 2007; 63: 151-4.

20. DE Paola M, DE Piano E, Pisani C, et al. Genital hypertrophicus lichen planus successfully treated with topical pimecrolimus. G Ital Dermatol Venereol 2018; 153: 296-8.

21. Simon D, Vassina E, Yousefi S, et al. Inflammatory cell numbers and cytokine expression in atopic dermatitis after topical pimecrolimus treatment. Allergy 2005; 60: 944-51.

22. Hengge UR, Krause W, Hofmann H, et al. Multicentre, phase II trial on the safety and efficacy of topical tacrolimus ointment for the treatment of lichen sclerosus. Br I Dermatol 2006; 155: 1021-8. 